\title{
Cross-Platform Social Web Application for Older Adults with HTML 5
}

\author{
Tiago Boldt Sousa ${ }^{1}$, Pedro Tenreiro ${ }^{1}$, Paula Alexandra Silva ${ }^{1}$, \\ Francisco Nunes ${ }^{1}$, and Eduarda Mendes Rodrigues ${ }^{2}$ \\ ${ }^{1}$ Fraunhofer Portugal, Rua Alfredo Allen 455/461 4200-135 Porto Portugal \\ ${ }^{2}$ Departamento de Engenharia Informática, Faculdade de Engenharia da \\ Universidade do Porto, Rua Dr. Roberto Frias, s/n 4200-465 Porto Portugal
}

\begin{abstract}
Online social networks can potentially play an important role in connecting older adults with family and friends who often live far. However, adoption of social Web services among the elderly is still very limited because the user interfaces are not adapted to them and also because few of their friends use such services. This paper introduces a mobile Web application designed with older adults' characteristics in mind that integrates a popular social network.
\end{abstract}

Keywords: storytelling, online social networks, user-centered interface design, older adults, html5.

\section{Introduction}

Older adults may face a reduction in personal contact mainly due to the loss of loved ones, mobility difficulties or geographical separation [10]. To keep in touch, families often use several communication channels such as mail, telephone and, more recently, online social networks. Although the first two are well mastered by older adults, the adoption of the latter is still low due to the complex user interfaces, which were not designed considering older adults' characteristics [10]. Nevertheless, social networks can potentially be a key for fighting loneliness and isolation among older adults, allowing them to stay connected with loved ones who may be physically distant [5].

This paper presents the design and implementation of a storytelling application for older adults, introducing the problems that older adults face in their daily life and their difficulties in using social networking services. The paper also describes the methods that are and have been used to design the application, their results and some details of the implementation.

\section{Use of Social Networks by Older Adults}

Older adults can find themselves in situations of social exclusion, due to retirement, moving difficulties or simply because they live far from friends and family [10]. A study with US elderly citizens reveals that most retirees are staying at home and onethird of those aged 75 and older live alone [9]. Loneliness may cause severe 
conditions, such as depression and insomnia [10]. This is due to the fact that, as human-beings we need to communicate with each other in order to share our knowledge and experiences $[1,2]$. Social exclusion is often aggravated by the onset of health conditions.

The use of ICTs (Information and Communication Technologies) is known to play a fundamental role in the psychological well-being of seniors, by enabling them to socialize, reducing feelings of loneliness and alienation [5]. However, the elderly struggle to adopt new technologies and software. This is partially due to the fact that the current generation of seniors grew up without computers and, therefore, have difficulties in perceiving the value and usefulness ICTs have in their daily activities [7]. But online social networks open up new and exciting opportunities for the social inclusion of older adults. In fact, they are giving more importance to this form of communication, acknowledging their benefits. According to Madden [6], the engagement of older adults in social media services in the US has doubled between April 2009 and May 2010 to 26\%. This study points out three main reasons for this increased adoption of social networks by older adults: i) reconnecting with people from their past; ii) seeking out advice a chronic diseases and sharing experience with others; iii) creating a bridge in generational gaps. Thus, older adults seem eager to start using social networking applications to communicate with friends and family but might be reluctant to do so due to the complex design of existing user interfaces. This calls upon careful design of future social Web applications, specifically intended at facilitating social inclusion of seniors.

\section{Designing a Social Web Application for Older Adults}

We are developing a social Web application for older adults, in which they should be able to communicate with friends and family to share their life-stories or general thoughts and feelings from their daily life. A user-centered design approach is fundamental to gather the needed information to start prototyping the design for such application. For this reason, we conducted informal interviews, observation, and usability tests in a day care center. These techniques were crucial to understand the needs, challenges and to obtain early feedback from the target audience.

\subsection{Sharing Life-Stories and Thoughts}

Storytelling presents advantages, not only for the storytellers themselves, but also for the story listeners. These stories carry life experiences to the next generations, the story listeners, establishing a bound between the two, providing them with wisdom that might influence their future [1,2]. A storytelling application for older adults that integrates with online social networks like Facebook, which is the most adopted social network world wide with more than 500 million users [4], has obvious advantages. Thus, the design of our application focuses primarily on the user experience addressing the characteristics of the elderly and leverages the Facebook API to post the user's stories and thoughts as wall posts. This enables standard Facebook users to post comments on the stories, which potentiates the interaction between the older adults and a large social network of contacts. 


\subsection{Results of the User-Centered Design Approach}

Usability tests with 25 subjects, with an average age of 75 , from a day care center showed that older adults struggle to perceive small sized items. Thus, items as text and images should be adapted to the user's ocular characteristics, adopting a size suitable for the user to read or watch the images with minimal effort. With the same usability tests we were able to determine that the minimum usable font size is of $2.8 \mathrm{~mm}$ so the older adults can read comfortably on mobile devices.

By carrying out informal interviews with the same set of older adults we confirmed findings described in the literature, observing that they get confused when shown large amounts of information, such as more than a message in the same display or even multiple notifications [3, 10]. When presented with many different items to process, older adults distract themselves easily. This might result in a loss of interest to use the application. With these interviews we concluded that it's important to reduce the application's complexity, by introducing only the essential features.

We also observed that all subjects from the day center had to listen or read questions or instructions more than once in order to understand them. Therefore, whenever showing information to older adults, we should always give them the necessary time to understand each piece of information, removing the possibility to use information that automatically disappears from the screen [3].

Older adults have higher rates of illiteracy than any other age bracket [8], hence proper images and sounds must exist to allow the replacement of the written text, so that illiterate seniors are able to use the system. It is relevant for older adults that our application allows them to record their voice or even a video to simplify the input process, removing the need for them to type text. With this information in mind, we know that we will need not only to stimulate the interest from seniors, but also to provide them with a pleasant and easy experience while using a social application. Therefore, our application ensures that the font size is appropriate for older adults, that there are no large amounts of information in each screen (e. g.: only a story each time) and that only the more important functionalities are present. This way they can perform their actions without getting confused with functionalities that they will not use such as events, groups or even external applications.

\subsection{Implementation}

The fast-paced growth of technology has led to the emergence of a number of different electronic devices. As the variety of devices grew, the technologies used to create software for them also expanded. HTML 5 aims to accelerate development by creating a Web standard that allows developers to create rich web applications that can be written once and that run in multiple devices, allowing users to choose the device that best suits them, considering portability, screen size, input method or even device costs. Because of the older adults' difficulties on adopting new technologies, this is an important feature for them to feel comfortable while trying the application. Thus, HTML 5 was used to implement an elderly-friendly storytelling application that seamlessly integrates with Facebook, enabling older adults to share stories with a potentially large social network of people that may be connected through any platform. 


\section{Conclusions}

Older adults are subject to feelings of loneliness resulting from isolation. Studies also show that older adults are receptive to adopting online social networks as a mean to improve their well-being and connect them with their acquaintances. With this in mind, our work provides a way for seniors to communicate with friends and family helping them to strength relationships and reduce feelings of loneliness. The application was designed to be as intuitive and pleasant as possible, adapting to common impairments and limitations of the older adult. HTML 5 allows the creation of a cross-platform application, giving the users the freedom to choose the device that best fits them.

\section{References}

1. Trentham, B.: Life storytelling, occupation, social participation and aging. Occupational Therapy Now, 23-26 (2007)

2. Sanders, B.: Reminiscence: Impacting brain fitness, lifelong learning, healthy aging \& wellness (2010)

3. Fisk, A.D., Rogers, W.A., Charness, N., Czaja, S.J., Sharit, J.: Designing for older adults: Principles and creative human factors approaches, 2nd edn., pp. 4200-8055. CRC Press, Boca Raton (2009) ISBN 978-1- 4200-8055-1

4. Wortham, J.: Facebook tops 500 million users, New York Times, p. B8 (2010)

5. Karavidas, M., Lim, N.K., Katsikas, S.L.: The effects of computers on older adult users. Computers in Human Behavior, 697-711 (2005)

6. Madden, M.: Older adults and social media social networking use among those ages 50 and older nearly doubled over the past year. Pew Research Center (2010)

7. Santana, P.C., Rodríguez, M.D., González, V.M., Castro, L.A., Andrade, A.G.: Supporting emotional ties among mexican elders and their families living abroad, pp. 2099-2103. ACM, New York (2005)

8. Roman, S.P.: Illiteracy and older adults: Individual and societal implications. Educational Gerontology 30(2), 79-93 (2004)

9. Steinfatt, T.: Social networks reduce loneliness and isolation for the elderly, http://www. homeinsteadrva.com/2010/07/19/social-networksreduce-loneliness-and-isolation-for-the-elderly/ (accessed January 2011)

10. Chen, Y.: Usability analysis on online social networks for the elderly (2009) 